\title{
Congenital cytomegalovirus (Cmv) infection and ascending aorta dilation
}

\begin{abstract}
Cytomegalovirus is the most frequent congenital infection worldwide and is diverse in its clinical manifestations. We report here a newborn baby who had congenital cytomegalovirus infection and was also found to have aortic root dilatation on echocardiography. We have noted 2 babies diagnosed with congenital CMV with incidental finding ascending aorta dilation, which completely resolved after adequate Ganciclovir treatment in one baby. Vertically transmitted HIV disease is known to cause arteriopathy and mild nonprogressive aortic root dilation in children between 2-9years of age, associated with increased serum HIV levels and lower CD4 counts. ${ }^{1}$ Ascending aorta dilation was a coincidental finding in these two newborns with congenital CMV disease, appearing at the time of birth and resolving by the end of 6weeks Gangciclovir treatment, We hypothesize that there is an association between Congenital CMV infection and dilatation of the ascending aorta.
\end{abstract}

Volume 4 Issue I - 2015

\author{
Tapas Mondal \\ Department of Pediatrics, McMaster University, Canada
}

Correspondence: Tapas Mondal, Associate Professor Pediatrics, division of Cardiology, McMaster University, I 200 Main Street West, room number 3A54, L8N3Z5 Hamilton, Ontario, Canada, Tel 9055212100, Fax 905521 I703, Email mondalt@mcmaster.ca

Received: November 14, 2015 | Published: November 23 2015

Keywords: ascending aorta dilatation, cytomegalovirus, congenital cmv infection and ascending aorta dilation

Abbreviations: CMV, cytomegalovirus; HIV, human immunodeficiency virus; VDRL, venereal disease research laboratories; IUGR, intrauterine growth retardation; PCR, polymerase chain reaction; NICU, neonatal intensive care unit; IVIG, intravenous immunoglobulin; PDA, patent ductusarteriosus; LV, left ventricle; LVEDD, left ventricular end diastolic dimension

\section{Introduction}

We present a case report on two neonates with congenital CMV and aortic dilation reversible after treatment with Gangciclovir Aortic dilation was a coincidental finding in these two newborns with multisystem involvement congenital CMV disease, appearing at the time of birth and resolving by the end of 6weeks treatment period after standard dosing of Valganciclovir, as seen in both our cases. We hypothesize that there is an association between Congenital CMV infection and dilation of the ascending aorta. Further research needs to be done to validate this association and the possible etiology as well as propose guidelines for long-term echocardiographic followup in survivors of congenital CMV disease. This is a novel report as we discovered after an extensive literature review, that the association between congenital CMV and aortic root dilation has not been previously published.

\section{Case presentation}

\section{Case I}

Baby boy A was born at 38weeks gestational age to a 20year old gravid mom. Pregnancy was uneventful. Mom was HIV negative, VDRL negative, HBsAg negative, Rubella immune and group B streptococcus positive. There was spontaneous onset of labour with rupture of membranes. He was delivered vaginally with Apgar scores of 4, 4 and 6 at 1, 5 and 10minutes respectively. He had no respiratory effort and was intubated. Birth weight was $3.16 \mathrm{~kg}$ (50th percentile) and head circumference was $35 \mathrm{~cm}$ (75th percentile). His size was appropriate for gestational age. Physical examination revealed jaundice, generalized petechiae and large hepatosplenomegaly. $\mathrm{He}$ was immediately started on intravenous antibiotics following a full septic workup. Laboratory investigations revealed a platelet count of 46,000, Urine culture was positive for Cytomegalovirus. Chest $\mathrm{X}$-Ray/Abdomen X-Ray revealed small right sided pleural effusion, hepatomegaly $7.8 \mathrm{~cm}$ and splenomegaly $8.8 \mathrm{~cm}$. Ultrasound of the head revealed calcification in the basal ganglia bilaterally and a $9 * 9 * 7$ $\mathrm{mm}$ cyst in the left temporal lobe adjacent to the temporal horn of the left lateral ventricle. Ophthalmology exam revealed bilateral retinal hemorrhages, but no chorioretinitis. Placental pathology showed features of chronic villitis with presence of plasma cells. Immunostaining in placental tissue was positive for CMV in 4 cells, but negative for Ebsteinbarr virus/ parvovirus/herpes virus 1 and 2 and toxoplasma. With findings suggestive of Congenital CMV infection, baby boy A was started on intravenous Gancyclovir. He was extubated on day 3 of life to low flow oxygen initially and then to room air. Antibiotics were discontinued after $48 \mathrm{hrs}$ of negative blood cultures. He was noted to have a murmur on physical exam. Echocardiogram revealed dilated ascending aorta with normal measurements of aortic valve annulus, aortic sinus and sinotubular junction. The supravalvular aorta was dilated ( $Z$ score 3.64 , absolute measurement of $1.2 \mathrm{~cm}$ ), with maximum dilation seen at the level of the ascending aorta $(\mathrm{Z}$ score 6). It was a normal aortic valve, baby had good biventricular function with no LV dilation. There was no flow acceleration across the trileaflet aortic valve. The baby had normal hemoglobin and was not tachycardiac or febrile at the time of the Echocardiogram (Figure 1). A follow up echocardiogram done at 6 months of age revealed partial resolution of the dilation of the supravalvular aorta $1.37 \mathrm{~cm} \mathrm{(Z}$ score 2.72 ), ascending aorta $1.16 \mathrm{~cm}$ ( $\mathrm{Z}$ score 2.29$)$.

\section{Case 2}

Baby Girl B was born at 30 weeks gestational age to a 32year old Gravida 4 mother. It was an uneventful pregnancy with maternal HIV, VDRL, Hepatitis B negative, Rubella Immune and Group B streptococcus not done due to early gestational age. Mum noted reduced fetal movements and non stress test was atypical with minimal beat to beat variability, suggesting significant fetal distress with no ultrasound evidence of hydrops. Baby was delivered by emergency Cesarean section after 2 adequate doses of antenatal Steroids. Baby had no initial respiratory efforts required stimulation and PPV for 5 minutes at $100 \%$ oxygen which was rapidly weaned off toroomair. 
Apgar scores were 7,8. At 1 minute and 5minute respectively. She was noted to have symmetric IUGR with both weight and head circumference less than $3 \mathrm{rd}$ centile (weight 1060grams and occipito frontal circumference $25 \mathrm{~cm}$ ).

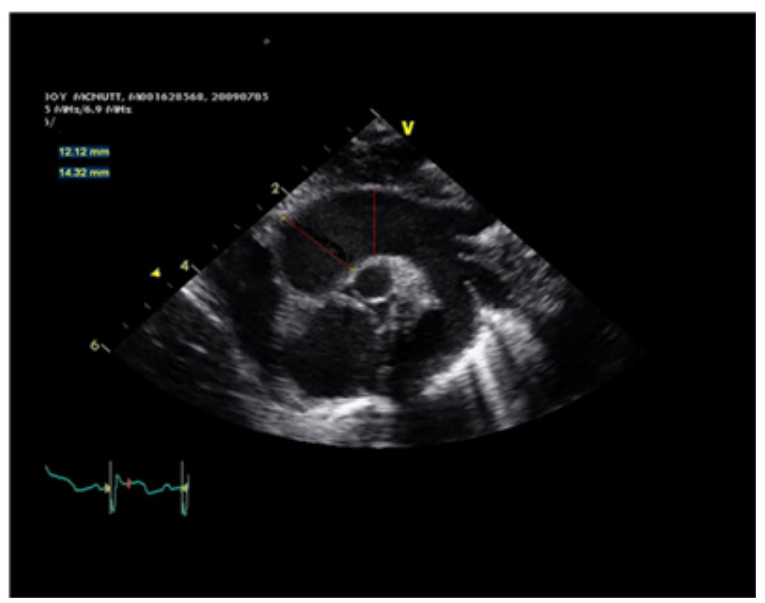

Figure I Echocardiogram.

Post natal events: Baby developed conjugated hyper bilirubinemia and neonatal hepatitis. Shehad persistant thrombocytopenia unresponsive to platelet transfusion (with generalised petechia baby received IVIG and daily platelet transfusions. Baby had normal hematocrit and hemoglobin levels, didnot require transfusions and remained hemodynamically stable. Urine CMV was positive at birth and placental histology and PCR was consistent with CMV infection. Parents refused lumbar puncture and CSF evaluation. Initial transfontanelle ultrasound showed bilateral grade 3 intraventricular hemorrhages with cystic changes suggestive of intrauterine bleed, no intracranial calcifications were seen. Baby had RDS (Respiratory distress syndrome) treated with CPAP till day 7 of life. She was found to have a moderate sized patent ductus arteriosus (PDA). Initial Echocardiogram the heart was structurally and functionally normal with minimal LV dilation. She was treated with indomethacin as per our NICU protocol for PDA closure, subsequent Echocardiogram showed closure of PDA, dilated ascending aorta $(7.8 \mathrm{~mm})$ with z score of 4.6 , the ascending aorta was dilated upto the isthmus which measured $5.3 \mathrm{~mm}$ ( $\mathrm{z}$ score 2.9 ), there was no evidence to suggest LV volume overload with normal LVEDD. There was no flow acceleration across the normal trileaflet aortic valve. Baby was started on IV Gangciclovir on day 4 of life for 2 weeks followed by oral Valganciclovir for additional 4weeks after consultation with our infectious disease team. Repeat Echocardiogram towards the end of the treatment regimen was completely normal with resolution of aortic dilation.

\section{Discussion}

Aortic root dilatation has been associated with: Bicuspid Aortic valve, 22q11.2 deletion, ${ }^{2}$ Marfan's syndrome, ${ }^{3}$ Ehler-Danlos syndrome, ${ }^{4}$ Turner syndrome, ${ }^{5}$ Noonan's syndrome, ${ }^{6}$ Post repair of Tetralogy of Fallot ${ }^{7}$, Syphilis, Ankylosing spondylitis, ${ }^{8}$ Hypertension and advanced age. ${ }^{9}$ Cytomegalovirus is a beta herpes virus with a double stranded DNA. CMV is the most frequent congenital infection worldwide and is diverse in its clinical manifestations. Congenital CMV infection occurs in approximately 0.2 to $2.5 \%$ of all live births. ${ }^{10}$ $10 \%$ of infants born with Congenital CMV infection show signs and symptoms at birth, ${ }^{11}$ the remaining $90 \%$ remain asymptomatic. The clinical features most commonly seen include petechiae/purpura, hepatosplenomegaly, jaundice, blueberry muffin spots, small for gestational age, microcephaly and periventricular calcifications. ${ }^{10}$
Other manifestations include seizures, intracranial hemorrhage, hydrocephalus, hypotonia, chorioretinitis, poor suck, high arched palate, sensorineural hearing loss, inguinal hernia, clubfoot, pneumonia, hepatitis and osteitis. ${ }^{10}$ There are no reports of cardiac structural abnormalities with Congenital CMV infection. The patients presented in our report have significant aortic root dilatation as evident by a Z score of 6.4. Repeat echocardiogram done at 6 months of age showed significant resolution of the Aortic root dilatation post therapy. This is not associated with changes in the LVEDD (left ventricular end diastolic diameter) suggesting volume overload caused by anemia or stress. In children with sickle cell anemia, increased left ventricular and aortic root dimensions have been reported to correlate well with hemoglobin levels. ${ }^{12}$ Examination and laboratory workup have ruled out other common causes of Aortic root and ascending aorta dilatation. Possible postulated causes for aortic root dilation in newborn clinically unwell with CMV disease include the following:

a. Stress modulated growth. ${ }^{13-15}$

b. Direct infection of the ascending aorta.

c. Systemic cytokine or lymphokine mediated alteration of the aortic root and ascending aorta

d. Central immune dysregulation as seen in other forms of immune mediated arteritis like ankylosing spondylitis and Takayasu's arteritis and Kawasaki's disease ${ }^{16-18}$ (List modified from Lai et al., $\left.{ }^{1}\right)$.

Aortic dilation was a coincidental finding in two newborns with multisystem involvement congenital CMV disease, appearing at the time of birth and resolving by the end of 6 weeks treatment period, as seen in both our cases. We hypothesize that there is an association between Congenital CMV infection and dilation of the ascending aorta. Further research needs to be done to validate this association and the possible etiology as well as propose guidelines for long-term echocardiographic follow-up in survivors of congenital CMV disease.

\section{Acknowledgements}

Dr. Rubeena Khan and Meenatchi Ranganathan compiled the report, Dr. Tapas Mondal provided access to the clinical information, provided expert advice and edited the report.

\section{Conflicts of interest}

Author declares there are no conflicts of interest.

\section{Funding}

None.

\section{References}

1. Lai WW, Colan SD, Easley KA, et al. Dilation of the aortic root in children affected with human immunodeficiency virus type 1: The prospective P2C2 HIV Multicenter Study. Am Heart J. 2001;141(4):661-670.

2. John AS, McDonald-McGinn DM, Zackai EH, et al. Aortic root dilation in patients with 22q11.2 deletion syndrome. Am J Med Genet A. 2009;149A(5):939-942.

3. Stuart AG, Williams A. Marfan's syndrome and the heart. Arch Dis Child . 2007;92(4):351-356.

4. Tiller GE, Cassidy SB, Wensel C, et al. Aortic root dilatation in EhlersDanlos syndrome types I, II and III - A report of five cases. Clin Genet . 1998;53(6):460-465.

5. Morgan T. Turner syndrome diagnosis and management. Am Fam Physician. 2007;76(3):405-410. 
6. Power PD, Lewin MB, Hannibal MC, et al. Aortic Root Dilatation is a Rare Complication of Noonan Syndrome. Pediatr Cardiol . 2006;27(4):478-480.

7. Tan JL, Gatzoulis MA, Ho SY. Aortic root disease in tetralogy of Fallot. Curr Opin Cardiol. 2006;21(6):569-572.

8. Tucker CR, Fowles RE, Calin A, et al. Aortitis in ankylosing spondylitis: early detection of aortic root abnormalities with two dimensional echocardiography. Am J Cardiol . 1982;49(4):680-686.

9. Cuspidi C, Meani S, Fusi V, et al. Prevalence and correlates of aortic root dilatation in patients with essential hypertension: relationship with cardiac and extracardiac target organ damage. $J$ Hypertens. 2006;24(3):573-580.

10. Leung AKC, Sauve RS, Davies HD. Congenital cytomegalovirus infection. J Natl Med Assoc. 2003;95(3):213-218.

11. Bar-Oz B, Berkovitch M, Ford-Jones L, et al. Canadian Family Physician. Motherisk update. 2001;47:1179-1181.

12. Lester LA, Sodt PC, Hutcheon N, et al. Cardiac abnormalities in children with sickle cell anemia. Chest . 1990;98(5):1169-1174.
13. Taber LA, Eggers DW. Theoretical Study of stress modulated growth in the aorta. $J$ TheorBiol. 1996;180(3):343-357.

14. Jones EC, Devereux RB, Michael JO'G, et al. Relation of hemodynamic volume overload to arterial and cardiac size. Journal of the American College of Cardiology. 1997;29(6):1303-1310.

15. Ascher G, Hohenadl $\mathrm{C}$, Schatz $\mathrm{O}$, et al. Infection with human immunodeficiency virus-1 increases expression of vascular endothelial growth factor in T Cells: implications of acquired immunodeficiency syndrome associated vasculopathy. Blood . 1999;93(12):4231-4241.

16. LaBresh KA, Lally EV, Sharma SC, et al. 2D Echocardiographic detection of preclinical aortic root abnormalities in rheumatoid variant disease. The American Journal of Medicine. 1985;78(6 Pt 1):908-912.

17. Brantley BD, Forman MB, Vimani R. Diagnosis and treatment of Takayasu's arteritis. Prim Cardiol. 1990;16:47-51,54.

18. Leung DY. The potential role of cytokine mediated vascular endothelial activation in the pathogenesis of Kawasaki Disease. Acta Paediatr Jpn. 1991;33(6):739-744. 